\title{
CHARACTERIZATIONS OF METRIC-DEPENDENT DIMENSION FUNCTIONS ${ }^{1}$
}

JAMES C. SMITH, JR.

1. Introduction. Let $(X, \rho)$ be a metric space, let $\operatorname{dim}(X)$ be the covering dimension of $X$, and let $d_{0}(X, \rho)$ be the metric dimension of $X$. Let $d_{2}$ and $d_{3}$ denote the metric-dependent dimension functions for metric spaces introduced by Nagami and Roberts [7], and let $d_{5}$ be the metric-dependent dimension function defined by Hodel [2]. A summary of the relationships among these dimension functions for $(X, \rho)$ is the following:

$$
d_{2}(X, \rho) \leqq d_{3}(X, \rho) \leqq d_{5}(X, \rho) \leqq d_{0}(X, \rho) \leqq \operatorname{dim}(X) \leqq 2 d_{3}(X, \rho) .
$$

In 1957, V. I. Egorov [1] characterized the dimension function $d_{0}$ in terms of Lebesgue covers as follows:

THEOREM. Let $(X, \rho)$ be a metric space. Then $d_{0}(X, \rho) \leqq n$ if and only if every Lebesgue cover of $X$ has an open refinement of order $\leqq n+1$.

In 1966, J. B. Wilkinson [9] similarly characterized the dimension function $d_{3}$.

Theorem. Let $(X, \rho)$ be a metric space. Then $d_{3}(X, \rho) \leqq n$ if and only if every finite Lebesgue cover of $X$ has an open refinement of order $\leqq n+1$.

In this paper we continue the study of Lebesgue characterization of metric-dependent dimension functions. In $\$ 2$ we give a Lebesgue cover characterization of $d_{2}$. In $\$ 3$ and $\$ 4$ we introduce two new metric-dependent dimension functions, $d_{6}$ and $d_{7}$, and characterize them in terms of Lebesgue covers.

Definition. Let $X$ be a set and $g=\left\{g_{\lambda}: \lambda \in \Lambda\right\}$ be a collection of collections of subsets of $X$. For each $\lambda \in \Lambda$, let $g_{\lambda}=\left\{G_{\alpha}: \alpha \in A_{\lambda}\right\}$. Then

$$
\bigwedge_{\lambda \in \Lambda}\left\{G_{\lambda}\right\}=\left\{\cap G_{\alpha(\lambda)}: \alpha(\lambda) \in A_{\lambda}, \lambda \in \Lambda\right\} .
$$

Definition. Throughout this paper $J$ will denote the set $\{1,2, \cdots, n+1\}$ and $J^{\prime}=J \cup\{n+2\}$, where the integer $n$ will always be understood.

Received by the editors August 22, 1967.

1 This work was taken from a doctoral dissertation at Duke University, and the research was supported in part by Langley Research Center (NASA) and NSF Grant GP-5919. I would like to thank Professors J. H. Roberts and R. E. Hodel for their many helpful suggestions while writing this paper. 
2. Characterization of $d_{2}$. The reader is referred to the papers by Nagami and Roberts [7] and by Hodel [2] for the definitions of the dimension functions $d_{0}, d_{2}, d_{3}$, and $d_{5}$. Note that in some papers $d_{0}$ and $\mu \operatorname{dim}$ are synonomous.

Definition 2.1. Let $\mathcal{G}=\left\{G_{\alpha}: \alpha \in A\right\}$ be a cover of a metric space $(X, \rho)$. We say that $\mathcal{G}$ is uniformly shrinkable if there exists a real number $\delta>0$ and a cover $\mathcal{F}=\left\{F_{\alpha}: \alpha \in A\right\}$ such that

(1) $F_{\alpha} \subset G_{\alpha}$ for all $\alpha \in A$.

(2) $\rho\left(F_{\alpha}, X-G_{\alpha}\right)>\delta$ for all $\alpha \in A$.

Theorem 2.2. Let $g$ be a cover of a metric space $(X, \rho)$. Then $G$ is a Lebesgue cover of $X$ if and only if $\mathcal{G}$ is uniformly shrinkable.

Proof (Necessity). Let $\mathcal{G}=\left\{G_{\alpha}: \alpha \in A\right\}$ be a Lebesgue cover of $(X, \rho)$ with Lebesgue number $\delta>0$. Define for each $\alpha \in A$,

$$
F_{\alpha}=\left\{x \in X: \rho\left(x,\left(X-G_{\alpha}\right)\right) \geqq \delta / 3\right\} .
$$

Clearly $F_{\alpha} \subset G_{\alpha}$ for all $\alpha \in A$. Let $x \in X$. Since $g$ is Lebesgue, $S(x, \delta / 2)$ $\subset G_{\beta}$ for some $\beta \in A$. Hence $x \in F_{\beta}$ so that $\mathcal{F}=\left\{F_{\alpha}: \alpha \in A\right\}$ covers $X$. Note that $\mathcal{F}$ is actually a Lebesgue cover, since $S(x, \delta / 6) \subset F_{\beta}$ above.

(Sufficiency). Suppose $\mathcal{G}=\left\{G_{\alpha}: \alpha \in A\right\}$ is uniformly shrinkable to $\mathcal{F}=\left\{F_{\alpha}: \alpha \in A\right\}$, where $\rho\left(F_{\alpha}, X-G_{\alpha}\right)>\delta$ for all $\alpha \in A$. Let $x \in X$. Since $\mathcal{F}$ covers $X, x \in F_{\beta}$ for some $\beta \in A$. Therefore, $S(x, \delta) \subset G_{\beta}$, and hence $\mathcal{G}$ is Lebesgue.

Construction Lemma. Let $X$ be a normal space, $\left\{G_{\alpha}: \alpha \in A\right\}$ a locally finite open collection, and $\left\{F_{\alpha}: \alpha \in A\right\}$ a closed collection such that $F_{\alpha} \subset G_{\alpha}$ for all $\alpha \in A$. If $\mathcal{G}=\bigwedge_{\alpha \in A}\left\{G_{\alpha}, X-F_{\alpha}\right\}$ has an open refinement of order $\leqq n+1$, then there exist closed sets $B_{\alpha}$ separating $F_{\alpha}$ and $X-G_{\alpha}$ for each $\alpha \in A$ such that ord $\left\{B_{\alpha}: \alpha \in A\right\} \leqq n$.

Proof. The proof proceeds essentially the same as the proof of $[8, \mathrm{II}, 5, \mathrm{~B}]$.

Theorem 2.3. Let $(X, \rho)$ be a metric space. Then $d_{2}(X, \rho) \leqq n$ if and only if for every collection $\left\{g_{i}: i \in J\right\}$ of $n+1$ binary Lebesgue covers of $X$, the cover $G=\Lambda_{i \in J} \mathcal{G}_{i}$ of $X$ has an open refinement of order $\leqq n+1$.

Proof (Necessity). Suppose $d_{2}(X, \rho) \leqq n$ and let $\mathcal{G}_{i}=\left\{G_{i}, X-F_{i}\right\}$, $i \in J$, be a collection of $n+1$ binary Lebesgue covers of $X$. Let $\mathcal{G}=\Lambda_{i \in J} \mathcal{G}_{i}$. We may assume each $\mathcal{G}_{i}$ to be an open cover by Theorem 2.2. It is clear that $\rho\left(F_{i}, X-G_{i}\right)>0$ for $i \in J$. Since $d_{2}(X, \rho) \leqq n$, there exist for each $i \in J$, open subsets $U_{i}$ of $X$ such that

(1) $F_{i} \subset U_{i} \subset\left(U_{i}\right)-\subset G_{i}$

(2) $\operatorname{ord}\left\{\left(\left(U_{i}\right)^{-}-U_{i}\right): i \in J\right\} \leqq n$. 
Define $W_{0}=\Lambda_{i \in J}\left\{U_{i}, X-\left(U_{i}\right)^{-}\right\}$. Clearly $\mathscr{W}_{0}$ satisfies

(1) $W_{0}$ covers $X-U_{i \in J} B_{i}$ where $B_{i}=\left(U_{i}\right)^{-}-U_{i}$.

(2) $W \in W_{0}$ implies there exists $G \in G$ such that $W \subseteq G$.

(3) $\operatorname{ord}\left(W_{0}\right) \leqq 1$.

(4) $W_{1}, W_{2} \in W_{0}$ implies $\left(W_{1}\right)^{-} \cap W_{2}=\varnothing=W_{1} \cap\left(W_{2}\right)^{-}$if $W_{1} \neq W_{2}$. Step 1. Define $J_{i}=J-\{i\}, J_{i, j}=J-\{i, j\}$, etc. Also let

$$
\varepsilon_{i}=\bigwedge_{j \in J_{i}}\left\{U_{j}, X-\left(U_{j}\right)^{-}\right\} \text {and } \mathcal{V}_{1}=\left\{B_{i} \cap E: E \in \mathcal{E}_{i}, i \in J\right\} \text {. }
$$

Note that $V_{1}$ is a partition of all points of order 1 with respect to $\left\{B_{i}: i \in J\right\}$. Also for different $V$ and $V^{\prime}$ in $V_{1}$ we have as in (4) above $\bar{V} \cap V^{\prime}=\varnothing=V \cap\left(V^{\prime}\right)^{-}$.

Since $X$ is completely normal and $v_{1}$ is finite, there exists a collection $W_{1}$ of pairwise disjoint open subsets of $X$ each containing one member of $\mathcal{V}_{1}$. Hence ord $\left(W_{1}\right) \leqq 1$, so that ord $\left(W_{0} \cup W_{1}\right) \leqq 2$. Also we may assume that $W \in W_{1}$ implies there exists a $G \in \mathcal{G}$ such that $W \subseteq G$. Otherwise, we intersect $W$ with $G$ to obtain this property.

Step 2. As in Step 1 define $\varepsilon_{i, j}=\Lambda_{k \in J_{i, j}}\left\{U_{k}, X-\left(U_{k}\right)^{-}\right\}$and $\mathcal{V}_{2}$ $=\left\{\left(B_{i} \cap B_{j}\right) \cap E: E \in \mathcal{E}_{i, j}, i, j \in J, i \neq j\right\}$. As before $v_{2}$ is a partition of all points of order 2 with respect to $\left\{B_{i}: i \in J\right\}$ such that for different $V$ and $V^{\prime}$ in $\mho_{2}$, we have again $\bar{V} \cap V^{\prime}=\varnothing=V \cap\left(V^{\prime}\right)^{-}$. Thus there exists a collection $W_{2}$ of pairwise disjoint open subsets of $X$ each containing one member of $\mathcal{V}_{2}$. Therefore, ord $\left(W_{2}\right) \leqq 1$, and hence $\operatorname{ord}\left(W_{0} \cup W_{1} \cup W_{2}\right) \leqq 3$. We may assume $W \subseteq G$ for every $W \in W_{2}$ and for some $G \in G$.

Now continue this process through step $n$, and define $\mathscr{W}=\cup_{i=0}^{n} W_{i}$. Since ord $\left\{B_{i}: i \in J\right\} \leqq n$, $W$ covers $X$. Also $W<\mathcal{G}$ and ord $(W) \leqq n+1$ by construction. Therefore $W$ is the desired open cover.

(Sufficiency). Let $\left\{C_{i}, C_{i}^{\prime}: i \in J\right\}$ be a collection of $n+1$ pairs of disjoint closed sets such that $\rho\left(C_{i}, C_{i}^{\prime}\right)>0$ for $i \in J$. Since $g_{i}$ $=\left\{X-C_{i}, X-C_{i}^{\prime}\right\}$ is a binary Lebesgue cover of $X, \mathcal{G}=\Lambda_{i \in J} g_{i}$ has a refinement of order $\leqq n+1$. By the Construction Lemma there exist closed sets $B_{i}$ separating $C_{i}$ and $C_{i}^{\prime}$ such that ord $\left\{B_{i}: i \in J\right\} \leqq n$. Hence $d_{2}(X, \rho) \leqq n$.

Theorem 2.4. Let $(X, \rho)$ be a metric space. Then $d_{2}(X, \rho) \leqq n$ if and only if every Lebesgue cover $\mathcal{G}=\left\{G_{1}, G_{2}, \cdots, G_{n+2}\right\}$ of $X$ consisting of $n+2$ members has an open refinement of order $\leqq n+1$.

Proof (Necessity). Suppose $d_{2}(X, \rho) \leqq n$, and let $G=\left\{G_{1}, G_{2}, \cdots\right.$, $\left.G_{n+2}\right\}$ be a Lebesgue cover of $X$ with Lebesgue number $\delta>0$. As in Theorem 2.2 define

$$
F_{i}=\left\{x \in X: \rho\left(x, X-G_{i}\right) \geqq \delta / 3\right\}
$$


for each $i \in J^{\prime}$, so that $\mathcal{F}=\left\{F_{1}, F_{2}, \cdots, F_{n+2}\right\}$ is a uniform shrink of g. Since $\left\{G_{i}, X-F_{i}\right\}$ is a Lebesgue cover of $X$ for $i \in J$, by Theorem $2.3 \mathcal{G}^{*}=\bigwedge_{i \in J}\left\{G_{i}, X-F_{i}\right\}$ has an open refinement $u$ such that $\operatorname{ord}(\mathcal{U}) \leqq n+1$. But $\mathcal{G}^{*}$ refines $\mathcal{G}$ since $\mathcal{F}$ covers $X$. Hence $\mathcal{u}$ is the desired open cover.

(Sufficiency). Let $\left\{C_{i}, C_{i}^{\prime}: i \in J\right\}$ be a collection of $n+1$ pairs of disjoint closed sets such that $\rho\left(C_{i}, C_{i}^{\prime}\right)=\delta_{i}>0$ for $i \in J$. Define $\delta=\min \left(\delta_{i}: i \in J\right), G_{i}=S\left(C_{i}, \delta / 2\right), H_{i}=\left(S\left(C_{i}, \delta / 4\right)\right)^{-}$for $i \in J$, and $G_{n+2}=X-\cup_{i \in J} H_{i}$. Since $\mathcal{G}=\left\{G_{1}, G_{2}, \cdots, G_{n+2}\right\}$ is a Lebesgue cover of $X$ by construction, $\mathcal{G}$ has an open refinement $\mathcal{U}=\left\{U_{\alpha}: \alpha \in A\right\}$ such that ord $(\mathcal{U}) \leqq n+1$. Let $f$ be the function, $f: A \rightarrow J^{\prime}$, defined by $f(\alpha)=$ smallest integer $i \in J^{\prime}$ such that $U_{\alpha} \subseteq G_{i}$. Now define $U_{i}=\bigcup\left\{U_{\alpha}: f(\alpha)=i\right\}$ for $i \in J^{\prime}$. Hence we may assume that $u$ $=\left\{U_{1}, U_{2}, \cdots, U_{n+2}\right\}$ with the order unchanged. Define

$$
E_{i}=\left\{x \in C_{i}: x \notin U_{i}\right\}, \quad S_{i}=S\left(E_{i}, \delta / 8\right), \quad V_{i}=U_{i} \cup S_{i}
$$

for $i \in J$, and $V_{n+2}=U_{n+2}$. Since $S_{i} \cap G_{n+2}=\varnothing$ for $i \in J$, then $v$ $=\left\{V_{1}, V_{2}, \cdots, V_{n+2}\right\}$ is an open cover of $X$ such that ord $(\mathcal{V}) \leqq n+1$ and $C_{i} \subset V_{i}$ for $i \in J$. Since $V$ is finite there exists a closed cover $\mathcal{F}=\left\{F_{1}, F_{2}, \cdots, F_{n+2}\right\}$ of $X$ such that $C_{i} \subseteq F_{i} \subseteq V_{i}$ for $i \in J^{\prime}[\mathbf{5}$, Lemma 1.5]. Thus $X$ normal implies there exist open sets $W_{i}$ such that $F_{i} \subset W_{i} \subset\left(W_{i}\right)^{-} \subset V_{i}$ for $i \in J$. Define $B_{i}=\left(W_{i}\right)^{-}-W_{i}$ for $i \in J$. Clearly $B_{i}$ separates $C_{i}$ from $C_{i}^{\prime}$ for $i \in J$. We assert $\bigcap_{i \in J} B_{i}=\varnothing$. Suppose there exists a point $x \in \bigcap_{i \in J} B_{i}$. Then $x \notin F_{i}$ for $i \in J$. Hence $x \in F_{n+2} \subset V_{n+2}$. But $x \in V_{i}$ for $i \in J$, so that $x \in \bigcap_{i=1}^{n+2} V_{i}$. This is a contradiction since ord $(V) \leqq n+1$. Hence $d_{2}(X, \rho) \leqq n$.

\section{The dimension function $d_{6}$.}

Definition 3.1. Let $(X, \rho)$ be a metric space. If $X=\varnothing, d_{6}(X, \rho)$ $=-1$. Otherwise, $d_{6}(X, \rho) \leqq n$ if $(X, \rho)$ satisfies this condition:

$\left(D_{6}\right)$ Given any countable collection of closed pairs $\left\{C_{i}, C_{i}^{\prime}\right.$ : $i=1,2, \cdots\}$ such that there exists $\delta>0$ with

(1) $\rho\left(C_{i}, C_{i}^{\prime}\right)>\delta$ for all $i$,

(2) $\left\{X-C_{i}^{\prime}: i=1,2, \cdots\right\}$ is locally finite,

then there exist closed sets $B_{i}$ separating $C_{i}$ from $C_{i}^{\prime}$ such that ord $\left\{B_{i}: i=1,2, \cdots\right\} \leqq n$. If $d_{6}(X, \rho) \leqq n$ is true and $d_{6}(X, \rho) \leqq n-1$ is false, then $d_{6}(X, \rho)=n$.

Note that $d_{6}(X, \rho) \leqq d_{5}(X, \rho)$ by definition.

Theorem 3.2. Let $(X, \rho)$ be a metric space. Then $d_{6}(X, \rho) \leqq n$ if and only if every countable, locally finite Lebesgue cover has an open refinement of order $\leqq n+1$.

Proof (Necessity). This proof is exactly the same as the proof of the necessity of Theorem 4.2 below and hence is omitted. 
(Sufficiency). Let $\left\{C_{i}, C_{i}^{\prime}: i=1,2, \cdots\right\}$ be a countable collection of closed pairs satisfying property $\left(D_{6}\right)$. Since $\left\{X-C_{i}^{\prime}\right.$ : $i=1,2, \cdots\}$ is locally finite, $\mathcal{G}=\Lambda_{i=1}^{\infty}\left\{X-C_{i}, X-C_{i}^{\prime}\right\}$ is a countable locally finite Lebesgue cover of $X$. Thus $\mathcal{G}$ has an open refinement of order $\leqq n+1$ and hence by the Construction Lemma, $d_{6}(X, \rho) \leqq n$.

Theorem 3.3. Let $(X, \rho)$ be a metric space. Every countable Lebesgue cover of $X$ has a countable locally finite Lebesgue refinement.

Proof. This proof is a modification of [2, Lemma 2.2]. Let $\mathcal{G}=\left\{G_{1}, G_{2}, \cdots\right\}$ be a Lebesgue cover of $X$ with Lebesgue number $\delta>0$. Define $F_{i}=\left\{x \in X: \rho\left(x, X-G_{i}\right) \geqq \delta / 2\right\}$ for all $i$. Then $\mathfrak{F}$ $=\left\{F_{1}, F_{2}, \cdots\right\}$ covers $X$ as before. Define $U_{i}=G_{i}-U_{j<i}\left(S\left(F_{j}, \delta / 8\right)\right)^{-}$ for all $i$, and $\mathcal{u}=\left\{U_{1}, U_{2}, \cdots\right\}$. Clearly $\mathcal{u}$ refines $\subseteq$ in a $1-1$ manner. We assert that $\mathcal{U}$ is a locally finite Lebesgue cover of $X$.

(1) Let $x \in X$. Choose the smallest $i$ such that $x \in\left(S\left(F_{i}, \delta / 8\right)\right)^{-}$. Then $x \in G_{i}-U_{j<i}\left(S\left(F_{j}, \delta / 8\right)\right)^{-}=U_{i}$. Hence $u$ covers $x$.

(2) Let $x \in X$. Choose the smallest $i$ such that $x \in S\left(F_{i}, \delta / 8\right)$. Then $S\left(F_{i}, \delta / 8\right) \cap U_{j}=\varnothing$ for $j>i$, so that $u$ is locally finite.

(3) Let $x \in X$. Choose the smallest $i$ such that $S(x, \delta / 8)$ $\cap\left(S\left(F_{i}, \delta / 8\right)\right)^{-} \neq \varnothing$. Then $S(x, \delta / 8) \subset G_{i}-U_{j<i}\left(S\left(F_{j}, \delta / 8\right)\right)^{-}=U_{i}$. Hence $\mathcal{U}$ is Lebesgue.

From Theorem 3.2 and Theorem 3.3 we have the following.

Theorem 3.4. Let $(X, \rho)$ be a metric space. Then $d_{6}(X, \rho) \leqq n$ if and only if every countable Lebesgue cover has an open refinement of order $\leqq n+1$.

Corollary 3.5. Let $(X, \rho)$ be a separable metric space. Then $d_{6}(X, \rho)=d_{0}(X, \rho)$.

Corollary 3.6 (Hodel). Let $(X, \rho)$ be a separable metric space. Then $d_{5}(X, \rho)=d_{0}(X, \rho)$.

4. The dimension function $d_{7}$.

Definition 4.1. Let $(X, \rho)$ be a metric space. If $X=\varnothing$, then $d_{7}(X, \rho)=-1$. Otherwise, $d_{7}(X, \rho) \leqq n$ if $(X, \rho)$ satisfies this condition:

$\left(D_{7}\right)$ Given any collection of closed pairs $\left\{C_{\alpha}, C_{\alpha}^{\prime}: \alpha \in A\right\}$ such that there exists $\delta>0$ with

(1) $\rho\left(C_{\alpha}, C_{\alpha}^{\prime}\right)>\delta$ for all $\alpha \in A$,

(2) $\left\{X-C_{\alpha}^{\prime}: \alpha \in A\right\}$ is locally finite,

then there exist closed sets $B_{\alpha}$ separating $C_{\alpha}$ and $C_{\alpha}^{\prime}$ such that ord $\left\{B_{\alpha}: \alpha \in A\right\} \leqq n$. If $d_{7}(X, \rho) \leqq n$ is true, and $d_{7}(X, \rho) \leqq n-1$ is false, then $d_{7}(X, \rho)=n$.

Theorem 4.2. Let $(X, \rho)$ be a metric space. Then $d_{7}(X, \rho) \leqq n$ if and only if every locally finite Lebesgue cover has a refinement of order $\leqq n+1$. 
Proof (Necessity). Suppose $d_{7}(X, \rho) \leqq n$, and let $\mathcal{G}=\left\{G_{\alpha}: \alpha \in A\right\}$ be a locally finite Lebesgue cover of $X$ with Lebesgue number $\delta>0$. By Theorem $2.2 \mathrm{G}$ is uniformly shrinkable to a closed cover $\mathcal{F}$ $=\left\{F_{\alpha}: \alpha \in A\right\}$ such that $F_{\alpha} \subset G_{\alpha}$ and $\rho\left(F_{\alpha}, X-G_{\alpha}\right) \geqq \delta / 3$ for all $\alpha \in A$. Since $d_{7}(X, \rho) \leqq n$ there exist closed sets $B_{\alpha}$ and open sets $U_{\alpha}$ and $U_{\alpha}^{\prime}$ which satisfy the following conditions:

(1) $B_{\alpha}$ separates $F_{\alpha}$ and $X-G_{\alpha}$ for all $\alpha \in A$.

(2) $X-B_{\alpha}=U_{\alpha} \cup U_{\alpha}^{\prime}$ for all $\alpha \in A$.

(3) $U_{\alpha} \cap U_{\alpha}^{\prime}=\varnothing$ for all $\alpha \in A$.

(4) ord $\left\{B_{\alpha}: \alpha \in A\right\} \leqq n$.

Since $X$ is paracompact there exist open sets $V_{\alpha}$ such that $B_{\alpha} \subset V_{\alpha}$ $C G_{\alpha}$ for all $\alpha \in A$, and ord $\left\{V_{\alpha}: \alpha \in A\right\} \leqq n$ [6, Theorem 1.3]. Let $\mathcal{V}=\left\{V_{\alpha}: \alpha \in A\right\}$. Define $\mathcal{U}=\bigwedge_{\alpha \in A}\left\{U_{\alpha}, U_{\alpha}^{\prime}\right\}$ which is a locally finite open cover of $X-\bigcup_{\alpha \in A} B_{\alpha}$, since $\mathcal{G}$ is locally finite. Also ord(น) $\leqq 1$, and $U \in \mathcal{U}$ implies that there exist some $G_{\beta} \in \mathcal{S}$ such that $U \subset G_{\beta}$.

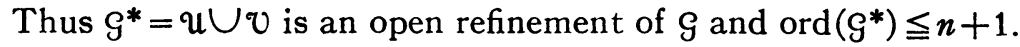

(Sufficiency). Suppose every locally finite Lebesgue cover of $X$ has an open refinement of order $\leqq n+1$. Let $\left\{C_{\alpha}, C_{\alpha}^{\prime}: \alpha \in A\right\}$ be any collection of closed pairs satisfying condition $\left(D_{7}\right)$ above. Then $\left\{X-C_{\alpha}, X-C_{\alpha}^{\prime}\right\}$ is a binary open Lebesgue cover of $X$ for each $\alpha \in A$. Since $\left\{X-C_{\alpha}^{\prime}: \alpha \in A\right\}$ is locally finite, $G=\Lambda_{\alpha \in A}\left\{X-C_{\alpha}, X-C_{\alpha}^{\prime}\right\}$ is a locally finite Lebesgue cover of $X$. Hence $g$ has an open refinement of order $\leqq n+1$. Therefore, by the Construction Lemma there exist closed sets $B_{\alpha}$ such that $B_{\alpha}$ separates $C_{\alpha}$ and $C_{\alpha}^{\prime}$ for each $\alpha \in A$ and ord $\left\{B_{\alpha}: \alpha \in A\right\} \leqq n$. Hence $d_{7}(X, \rho) \leqq n$.

\section{REFERENCES}

1. V. I. Egorov, On the metric dimension of point sets, Mat. Sb. 48 (1959), 227-250.

2. R. E. Hodel, Note on metric-dependent dimension functions, Fund. Math. 61 (1967), 83-89.

3. W. Hurewicz and H. Wallman, Dimension theory, Princeton Univ. Press, Princeton, N. J., 1955.

4. M. Katětov, On the relation between metric and topological dimensions, Czechoslovak. Math. J. 83 (1958), 163-166. 5-36.

5. K. Morita, On the dimension of normal spaces. I, Japan. J. Math. 20 (1950), 33.

6. - - On the dimension of normal spaces. II, J. Math. Soc. Japan 2 (1950), 16-

7. K. Nagami and J. H. Roberts, A study of metric-dependent dimension functions, Trans. Amer. Math. Soc. 129 (1967), 414-435.

8. J. Nagata, Modern dimension theory, Interscience, New York, 1965.

9. J. B. Wilkinson, Covering dimension and metric-dependent dimension functions, Ph.D. dissertation, Duke University, Durham, N.C., 1966.

Virginia Polytechnic Institute 